\title{
Fatores de risco para morte por pneumonia em menores de um ano em uma região metropolitana do sudeste do Brasil. Um estudo tipo caso-controle*
}

\author{
Risk factors for death by pneumonia among children under one year of \\ age in a metropolitan area of Southeastern Brazil. A case-control study
}

Flávia Maria Leal Niobey**, Milena Piraccini Duchiade**, Ana Glória Godoi Vasconcelos***, Márcia Lázaro de Carvalho**, Maria do Carmo Leal ${ }^{\star \star}$, Joaquim Gonçalves Valente

\begin{abstract}
NIOBEY, F.M.L. et al. Fatores de risco para morte por pneumonia em menores de um ano em localidade urbana na região sudeste do Brasil. Um estudo tipo caso-controle. Rev. Saúde públ., S.Paulo, 26: 229-38, 1992. Através de um estudo tipo caso-controle, foi comparada uma amostra de óbitos pós-neonatais por pneumonia ocorridos na Região Metropolitana do Rio de Janeiro, Brasil (1986-1987) e controles sadios, moradores na vizinhança. Os fatores de risco investigados foram variáveis relacionadas à história gestacional da mãe e ao nascimento da criança, às condições sociais da famllia e à utilizacão de serviços de saúde. $\mathrm{Na}$ primeira etapa de análise, através de um modelo de regressão logística univariada, foram estimados os coeficientes de cada variável independente, o risco relativo e seus limites de confiança. $O$ peso ao nascer e a idade do desmame mostraram-se das mais fortemente associadas com a variável dependente. Na segunda etapa, foi feito o ajuste pelo modelo de regressão logística múltipla e somente 4 variáveis permaneceram estatisticamente associadas com a mortalidade: idade do desmame, peso ao nascer, número de moradores da casa e aplicação da vacina BCG. Concluise que a mortalidade por pneumonia em menores de um ano está fortemente associada às condições sociais da familia, em particular da mãe.
\end{abstract}

Descritores: Pneumonia, mortalidade. Mortalidade infantil. Fatores de risco.

\section{Introdução}

As Infecçōes Respiratórias Agudas (IRAs) constituem a principal causa de adoecimento em crianças até 5 anos de idade, tanto nos países desenvolvidos quanto nas regiões em desenvolvimento. Diversos estudos longitudinais constataram que o número médio de episódios de IRAs em crianças moradoras de áreas urbanas variava entre 4,2 e 7,9 episódios por ano e entre 1 e 3 naquelas que viviam em zonas rurais ${ }^{26}$. Investigações desenvolvidas no Brasil chegaram a conclusões semelhantes,

* Financiado pela Fundação de Amparo à Pesquisa do Estado de São Paulo. Processo $n^{2} 43860197$. OC.

* Departamento de Epidemiologia da Escola Nacional de Saúde Pública (FIOCRUZ) e da Secretaria Municipal de Saúde - Rio de Janeiro, RJ - Brasil.

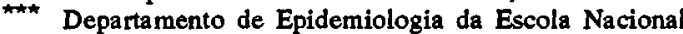
de Saúde Pública (FIOCRUZ) - Rio de Janeiro, RJ Brasil.

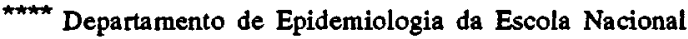
de Saúde Pública (FIOCRUZ) e do Instituto de Medicina Social da Universidade Estatual do Rio de Janeiro - Rio de Janeiro, RJ - Brasil.

Separatas/Reprints: F.M.L. Niobey - R Leopoldo Bulhôes, 1480 - $3^{2}$ andar - 21041-210 - Rio de Janeiro, RJ - Brasil. quanto à importância das IRAs na morbidade de lactentes e pré-escolares ${ }^{16,19,20}$.

Embora as IRAs se manifestem em geral sob formas benignas, alguns grupos etários são particularmente vulneráveis às suas complicações, das quais a mais temida é a pneumonia; sabe-se de longa data que epidemias de gripe provocam excessos de mortalidade nos maiores de 65 anos, tanto devido às pneumonias quanto por outras causas ${ }^{32}$. Os lactentes, devido ao desenvolvimento incompleto do aparelho pulmonar, constituem outro grupo especialmente predisposto ${ }^{3}$, sendo que o impacto das IRAs, enquanto agravo à saúde, varia conforme o nivel socioeconômico das populações afetadas. Assim, embora a incidência das infecções respiratórias possa ser bastante semelhante nas diferentes regiōes, a mortalidade por doenças respiratórias adquire especial relevância nos países em desenvolvimento ${ }^{5,15,25,26,29}$.

No Brasil, que apresenta taxas elevadas de mortalidade infantil da ordem de 60 por mil, bem superiores à de países vizinhos, com nivel de desenvolvimento econômico similar 
ou mesmo inferior ${ }^{35}$, as pneumonias (Classificação Internacional de Doenças - CID - IX: 480-486) constituem a terceira causa de mortalidade infantil, vindo atrás das afecções perinatais (cap. XV da CID) e das diarréias (CID IX: 001-009) ${ }^{18}$. Entretanto, a estrutura de causas apresenta características diferentes conforme as Regiōes. Assim, apesar das afecções perinatais terem sido responsáveis por $45,8 \%$ do total de óbitos em menores de um ano com causa conhecida em 1985 e desse quadro repetir-se em praticamente todos os Estados, as diarréias tendem à queda, devido à melhora paulatina do sistema de abastecimento de água, o que leva as pneumonias a assumirem o segundo lugar, em Estados como São Paulo e Rio de Janeiro ${ }^{18}$.

Como as afecçōes perinatais concentramse no período neonatal (até os 27 dias de vida), temos então que as pneumonias já são a principal causa de morte no período pósneonatal, em alguns Estados, como os já citados. No Estado do Rio de Janeiro, entre 1977 e 1986, as pneumonias, apesar de apresentarem taxas em declínio, já substituíram as diarréias, enquanto segundo causa de morte entre os menores de um $a^{34}{ }^{34}$.

A crescente consciência a respeito da importância das doenças respiratórias agudas tem levado as autoridades sanitárias, já desde meados da década de 80 , a incluírem o programa de controle das IRAs nas Ações Básicas para Assistência Integral à Saúde da Criança. Estes programas, baseados na definição de "padrões de procedimentos para diagnóstico e terapêutica, que orientam condutas diferenciadas segundo a gravidade do caso e o grau de complexidade dos serviços de saúde ${ }^{\prime \prime} 17$, esbarram em problemas cuja raiz reside, de um lado, no precário funcionamento da rede básica de saúde, já constatado, e, de outro, na resistência que os profissionais de saúde, em especial os médicos, demonstram para se submeter às condutas ditas "normatizadas".

A ênfase dos programas de combate às IRAs recai nas medidas ditas de prevenção secundária, a começar pelas propostas da Organização Mundial de Saúde. As normas atualmente em vigor mencionam apenas brevemente algumas açōes de prevenção primária, que se resumem em sintese à necessidade das imunizaçōes obrigatórias, e à recomendação para "evitar que a criança fique em ambientes poluidos por fumaça causada por fogo ou cigarro"17.

Dentre os fatores predisponentes, têm sido apontados a desnutrição ${ }^{14}$, o desmame precoce $^{38}$ e um conjunto agrupado genericamente sob a denominação de "condições de vida", que envolvem desde o nível socioeconômico da familia, a idade e a instrução da mãe, até a densidade de moradores por domicílio, entre outros ${ }^{3}$. Dentre os "critérios prognósticos de gravidade", o manual de Ministério da Saúde ${ }^{17}$ (1984) ressalta a idade (recémnatos ou lactentes), o baixo peso ao nascer, a desnutrição, em especial a de grau III, a duração da doença e a presença de patologias associadas, tais como a diarréia, o sarampo ou outras.

A associação entre condições socioeconômicas e os níveis de mortalidade por doenças respiratórias é conhecida de longa data ${ }^{39}$, tendo sido confirmada por estudos recentes ${ }^{8,30}$. Divcrsos autores têm destacado a importância do tamanho da família, medido seja pela existência de irmãos, seja pela densidade de moradores - por domicílio ou por cômodo - na transmissão de doenças respiratórias, como a tuberculose, as IRAs ou o sarampo, devido à maior oportunidade de contatos $1,8,12,39$. O mesmo mecanismo explicaria a maior proporção de infecções respiratórias alta e/ou otites nas crianças menores de cinco anos que freqüentam creches ${ }^{2,10}$.

O objetivo do presente trabalho é identificar os fatores de risco sociais e biológicos que contribuem para elevar o risco de morrer por pneumonias em menores de um ano em nosso meio. Numa segunda etapa, serão analisadas algumas características da atenção médica prestada, que podem estar interferindo para aumentar a chance de morte.

\section{Material e Método}

O presente trabalho é um desdobramento do projeto "Determinantes da Mortalidade Infantil na Regiāo Metropolitana do Rio de Janeiro - maio/86 a abril/87", cujos primeiros resultados já foram divulgados ${ }^{6,7,9,23}$.

O trabalho de campo dividiu-se em visitas domiciliares e hospitalares. As hospitalares foram realizadas por médicos e consistiam no preenchimento de um questionário com dados do prontuário da criança falecida (os "casos"). Além disso, eram preenchidos questionárioscontroles de crianças que tiveram alta ("controles hospitalares"). Os critérios de seleção do controle foram: a causa da internação diarréia, pneumonia ou causas perinatais - o grupo etário (menor de 29 dias, 29 dias a 5 
meses, 6 meses e mais), e a época de permanência no mesmo hospital do caso.

As visitas domiciliares foram realizadas por visitadores sanitários treinados. Para cada óbito pelas 3 causas citadas foi selecionado um "controle sadio" da mesma faixa etária e morador no quarteirão do caso sorteado. Foram assim colhidas informações com as mães (ou com outro responsável adulto), dos "casos" e dos "controles" (hospitalares e sadios) através de um questionário especifico.

Após a coleta dos dados hospitalares, a equipe preenchia um novo atestado de óbito, com base nas informações do prontuário, obedecendo aos critérios recomendados pelo Centro Brasileiro de Classificação de Doen$\operatorname{ças}^{24}$.

Os óbitos foram ainda classificados, segundo o "local de ocorrência" constante da Declaração de Óbito, em: extra-hospitalares (domicílio, via pública, delegacia e outros) e hospitalares. Verificou-se, porćm, que alguns óbitos dados como hospitalares, na verdade haviam ocorrido antes de a ctiança dar entrada no serviço de saúde, motivo pelo qual passaram a fazer parte do grupo de extra-hospitalares. Por não possuir prontuário, esse grupo só apresenta dados do questionário domiciliar. Outros detalhes sobre a metodologia podem ser encontrados nos trabalhos supracitados.

Por tratar-se de estudo caso-controle, o grupo de "casos" é formado pelos óbitos pósneonatais da amostra, codificados originalmente como devidos à pneumonia (CID-IX : 480-486), e confirmados pelo prontuário, além dos que passaram a ser atribuídos às pneumonias, após preenchimento do novo atestado.

Os óbitos até 28 dias não foram considerados devido ao pequeno número de mortes por pneumonia nesta faixa (seis). Os óbitos que deixaram de ter como causa básica pneumonia, após revisão dos prontuários, foram excluídos. Os óbitos "extra-hospitalares" atribuídos às pneumonias foram conservados no grupo de "casos", mesmo sem confirmação por necrópsia, uma vez que representavam percentual ponderável do total $(43,3 \%)$. Foram incluídos também, os controles hospitalares e sadios selecionados a partir dos casos; se não houvesse perdas, existiriam dois tipos de controle para cada caso. Nesta análise, foram considerados apenas os casos e controles sadios, ficando para uma etapa posterior a comparação entre casos e controles hospitalares.

A composição da subamostra, após as devidas correções, encontra-se na Tabela 1.
Tabela 1. Composição da subamostra estudada.

\begin{tabular}{lcc}
\hline $\begin{array}{l}\text { Composição } \\
\text { da subamostra }\end{array}$ & $\begin{array}{c}\text { Questionários } \\
\text { Hospitalares }\end{array}$ & $\begin{array}{c}\text { Questionários } \\
\text { Domiciliares }\end{array}$ \\
\hline Casos & $200^{*}$ & $255^{* *}$ \\
Controles Hosp. & 170 & 116 \\
Controles sadios & $\ldots$ & $239^{* * *}$ \\
\hline
\end{tabular}

- Do total de 363 casos, 157 foram extra-hospita. lares e 6 foram perda.

** Do total de 363 casos, $108(29,7 \%)$ foram perda de questionário domiciliar, em sua maioria por não localização do endereço ou mudança.

*** Em 16 casos, não foram obtidos controles na vizinhança

A distribuição etária dos casos e controles sadios era semelhante, com idade mediana de 117,5 dias e 130,0 dias respectivamente.

$\mathrm{Na}$ primeira parte do presente estudo foram identificados os fatores de risco (variáveis independentes) associados à morte por pneumonia, considerando apenas os casos (óbitos) e os controles sadios. A variável resposta ficou definida como: - 1: morte por pneumonia. - 0: criança sadia.

As variáveis independentes foram classificadas em 3 grupos, conforme proposta adaptada de Mosley ${ }^{21}$ :

Grupo I - Fatores relativos à história gestacional da mãe e ao nascimento da criança:

a) idade da criança: variável contínua (IDADEC)

b) peso da criança ao nascer: variável contínua (PESNASC)

c) fumo na gestação da criança em questão: "sim" ou "não" (FUMGEST)

d) ordem de nascimento da criança: "Quarto filho em diante" contra "até terceiro filho" (ORDNASC)

e) idade da mãe: "14-19 anos/35-49 anos" contra 20-35 anos" (IDAMAE)

f) idade do desmame, definida em adequada ou não (DESMAME):

Casos - risco: se deixou de mamar antes de ser internado ou antes de 6 meses; não risco: se mamou até ser internado ou durante 6 meses ou mais.

Controles - risco: se deixou de mamar antes de 6 meses;

não risco: se ainda mamava ou se mamou durante 6 meses ou mais. 
Grupo II - Fatores relativos às condições sociais da familia:

a) grau de instrução dos pais: "analfabeto ou sabendo ler e escrever" contra "pelo menos primário completo" (INSTPAI e INSTMAE)

b) renda familiar "per capita": "menos de 0,5 salários-mínimos", contra "0,5 e mais" (RENDFPC)

c) presença de fumantes no domicílio: "um fumante e mais", contra "nenhum fumante" (PMORFUM)

d) número de moradores: "5 ou mais moradores" contra "até 4 moradores" (NMORAD)

\section{Grupo III - Fatores relativos à utilização de} serviços de saúde:

a) vacina BCG: "não tomou", contra "tomou" (BCG)

b) história prévia de internação por qualquer causa: "uma e mais internações", contra "nenhuma internação" (NINTERN)

c) história prévia de asma brônquica e/ou pneumonia: "teve", contra "não teve" (DOENÇA).

Utilizou-se inicialmente, o teste de independência qui-quadrado para selecionar as variáveis relacionadas com o risco de adoecer e morrer por pneumonia $(\mathrm{p}<0,05)$. Os resultados da aplicação deste teste e a distribuição (\%) de casos e controles nas categorias de tisco encontram-se na Tabela 2.

$O$ peso ao nascer foi trabalhado como variável contínua, devido ao tipo de distribuição dessa variável na amostra.

Para analisar o efeito das variáveis con-

Tabela 2 . Distribuição (\%) de casos e controles nas categorias de risco, qui-quadrado e nivel de significân. cia para as variáveis incluídas no estudo

\begin{tabular}{|c|c|c|c|c|}
\hline Variável* & Casos & Controles & Qui-quadrado & $\mathbf{P}$ \\
\hline FUMGEST & 59,80 & 40,20 & 8,70 & 0,003 \\
\hline ORDNASC & 66,19 & 33,81 & 16,21 & 0,000 \\
\hline IDMAE & 53,72 & 46,28 & 0,432 & 0,511 \\
\hline DESMAME & 64,92 & 35,08 & 66,13 & 0,000 \\
\hline INSTMAE & 60,85 & 39,15 & 11,63 & 0,001 \\
\hline INSTPAI & 58,39 & 41,61 & 7,23 & 0,007 \\
\hline RENDFPC & 55,72 & 44,28 & 3,88 & 0,049 \\
\hline PMORFUM & 55,03 & 44,97 & 6,18 & 0,013 \\
\hline NMORAD & 60,77 & 39,23 & 26,74 & 0,000 \\
\hline$B C G$ & 67,76 & 32,24 & 24,85 & 0,000 \\
\hline NINTERN & 80,00 & 20,00 & 22,93 & 0,000 \\
\hline DOENCA & 79,41 & 20,59 & 24,93 & 0,000 \\
\hline
\end{tabular}

- Ver no texto o significado das siglas. juntamente, utilizou-se o pacote estatístico MULTLR ("multiple logistic regression") para o modelo de regressão logística múltipla. O critério utilizado para a seleção das variáveis na composição do modelo final foi baseado nos coeficientes de correlação de Pearson observados para cada grupo de variáveis (Tabela 3), no nível de significância das estatísticas apresentadas na análise univariada (qui-quadrado e intervalo de confiança para o "odds ratio") (Tabela 2,4), e na aplicação da regressão multivariada para cada grupo isoladamente (Tabela 5).

\section{Resultados}

A análise dos dados foi dividida em etapas. Numa primeira fase foi feita uma análise univariada, onde foram estimados os coeficientes de cada variável independente, o risco relativo e seus limites de confiança. A partir desses resultados (Tabela 4), pode-se considerar, separadamente, cada variável enquanto fator de risco para morte por pneumonia; a única exceção foi a variável "idade da mãe" por sua baixa associação com a variável resposta $(p>0,05)$.

Numa segunda etapa, foi aplicado o modelo de regressão multivariada por grupo de classificação (Grupos I e II), buscando determinar as variáveis mais relevantes ou mais fortemente associadas com a morte por pneumonia, dentro de cada grupo (Tabela 5). Estas vieram a constituir o modelo multivariado final, adicionando-se a variável BCG do Grupo III.

Os resultados da fase univariada encontram-se na Tabela 4. Dentre as variáveis, o peso ao nascer e idade do desmame mostraram-se das mais fortemente associadas com a variável dependente. $O$ coeficiente negativo do PESNASC estabelece uma relação inversa com esta, ou seja, um maior peso ao nascer diminui a chance de morrer por pneumonia. Sendo assim, o risco relativo menor que um leva à caracterização como fator de proteção. A unidade de variação considerada na sua análise é $100 \mathrm{~g}$; dessa forma, para cada $100 \mathrm{~g}$ mais de peso, diminui a chance relativa de a criança morrer por pneumonia em $36 \%$.

Ainda nesta fase, as variáveis "história prévia de internação" e "história prévia de doença (asma e/ou pneumonia)", embora apresentassem valores altos para o risco relativo estimado $(4,45$ e 4,38$)$, mostraram também in- 
Tabela 3 . Matriz de correlação para os fatores de risco: coeficiente de correlação de Pearson e nivel de significância

\begin{tabular}{|c|c|c|c|c|c|c|c|c|c|c|c|c|c|}
\hline $\begin{array}{l}\text { Fatores } \\
\text { de risco" }\end{array}$ & IDADEC & DESMAME & PESNASC & FUMGEST & ORDNASC & INSTMAE & INSTPAI & RENDFPC & PMORFUM & NMORAD & $B C G$ & DOENÇA & NINTERN \\
\hline IDADEC & $\begin{array}{l}1,00000 \\
0,0\end{array}$ & & & & & & & & & & & & \\
\hline DESMAME & $\begin{array}{l}0,10552 \\
0,0201\end{array}$ & $\begin{array}{l}1,00000 \\
0,0\end{array}$ & & & & & & & & & & & \\
\hline PESNASC & $\begin{array}{l}0,07469 \\
0,1081\end{array}$ & $\begin{array}{l}0,04148 \\
0,3758\end{array}$ & $\begin{array}{l}1,00000 \\
0,0\end{array}$ & & & & & & & & & & \\
\hline FUMGEST & $\begin{array}{l}0,01393 \\
0,7573\end{array}$ & $\begin{array}{l}0,11784 \\
0,0095\end{array}$ & $\begin{array}{l}-0,17922 \\
0,0001\end{array}$ & $\begin{array}{l}1,00000 \\
0,0\end{array}$ & & & & & & & & & \\
\hline ORDNASC & $\begin{array}{l}0,00383 \\
0,9323\end{array}$ & $\begin{array}{l}0,04274 \\
0,3481\end{array}$ & $\begin{array}{l}0,00198 \\
0,9661\end{array}$ & $\begin{array}{l}0,10195 \\
0,0233\end{array}$ & $\begin{array}{l}1,00000 \\
0,0\end{array}$ & & & & & & & & \\
\hline INSTMAE & $\begin{array}{l}0,00490 \\
0,9141\end{array}$ & $\begin{array}{l}0,00605 \\
0,8952\end{array}$ & $\begin{array}{c}-0,05791 \\
0,2171\end{array}$ & $\begin{array}{l}0,03075 \\
0,4988\end{array}$ & $\begin{array}{l}0,23065 \\
0,0001\end{array}$ & $\begin{array}{l}1,00000 \\
0,0\end{array}$ & & & & & & & \\
\hline INSTPAI & $\begin{array}{l}0.10890 \\
0.0343\end{array}$ & $\begin{array}{l}0,01342 \\
0,7966\end{array}$ & $\begin{array}{c}-0,00394 \\
0,9406\end{array}$ & $\begin{array}{l}0,07150 \\
0,1659\end{array}$ & $\begin{array}{l}0,29659 \\
0,0001\end{array}$ & $\begin{array}{l}0,46937 \\
0,0001\end{array}$ & $\begin{array}{l}1,00000 \\
0,0\end{array}$ & & & & & & \\
\hline RENDFPC & $\begin{array}{l}0,00032 \\
0,9944\end{array}$ & $\begin{array}{c}-0,05496 \\
0,2339\end{array}$ & $\begin{array}{l}0,10109 \\
0,0319\end{array}$ & $\begin{array}{l}0,01858 \\
0,6847\end{array}$ & $\begin{array}{l}0,29450 \\
0,0001\end{array}$ & $\begin{array}{l}0,28431 \\
0,0001\end{array}$ & $\begin{array}{l}0,28984 \\
0,0001\end{array}$ & $\begin{array}{l}1,00000 \\
0,0\end{array}$ & & & & & \\
\hline PMORFUM & $\begin{array}{l}0,04354 \\
0,3337\end{array}$ & $\begin{array}{l}0,05889 \\
0,1959\end{array}$ & $\begin{array}{l}0,09807 \\
0,0349\end{array}$ & $\begin{array}{l}0,35076 \\
0,0001\end{array}$ & $\begin{array}{l}0,08387 \\
0,0625\end{array}$ & $\begin{array}{l}0,13972 \\
0,0020\end{array}$ & $\begin{array}{l}0,12503 \\
0,0151\end{array}$ & $\begin{array}{l}0,14181 \\
0,0018\end{array}$ & $\begin{array}{l}1,00000 \\
0,0\end{array}$ & & & & \\
\hline NMORAO & $\begin{array}{l}0,03778 \\
0,4012\end{array}$ & $\begin{array}{l}0,05808 \\
0,2016\end{array}$ & $\begin{array}{c}-0,02988 \\
0,5209\end{array}$ & $\begin{array}{l}0,08835 \\
0,0495\end{array}$ & $\begin{array}{l}0,36192 \\
0,0001\end{array}$ & $\begin{array}{l}0,11328 \\
0,124\end{array}$ & $\begin{array}{l}0,09449 \\
0,0665\end{array}$ & $\begin{array}{l}0,23285 \\
0,0001\end{array}$ & $\begin{array}{l}0,13045 \\
0,0036\end{array}$ & $\begin{array}{l}1,00000 \\
0,0\end{array}$ & & & \\
\hline$B C G$ & $\begin{array}{l}0,29101 \\
0,0001\end{array}$ & $\begin{array}{l}0,00964 \\
0,8344\end{array}$ & $\begin{array}{c}-0,18422 \\
0,0001\end{array}$ & $\begin{array}{l}0,03951 \\
0,3873\end{array}$ & $\begin{array}{l}0,20967 \\
0,0001\end{array}$ & $\begin{array}{l}0,13403 \\
0,0035\end{array}$ & $\begin{array}{l}0,14216 \\
0,0062\end{array}$ & $\begin{array}{l}0,11816 \\
0,0105\end{array}$ & $\begin{array}{l}0,07459 \\
0,1023\end{array}$ & $\begin{array}{l}0,13352 \\
0,0033\end{array}$ & $\begin{array}{l}1,00000 \\
0,0\end{array}$ & & \\
\hline DOENÇA & $\begin{array}{l}0,14115 \\
0,0017\end{array}$ & $\begin{array}{l}0,08633 \\
0,0580\end{array}$ & $\begin{array}{c}-0,05015 \\
0,2821\end{array}$ & $\begin{array}{l}0,03374 \\
0,4562\end{array}$ & $\begin{array}{l}-0,02470 \\
0,5855\end{array}$ & $\begin{array}{l}0,04993 \\
0,2736\end{array}$ & $\begin{array}{l}0,09321 \\
0,0710\end{array}$ & $\begin{array}{l}0,01556 \\
0,7350\end{array}$ & $\begin{array}{c}-0,02641 \\
0,5597\end{array}$ & $\begin{array}{l}0,01472 \\
0,7449\end{array}$ & $\begin{array}{l}0,07015 \\
0,1248\end{array}$ & $\begin{array}{l}1,00000 \\
0,0\end{array}$ & \\
\hline NINTERN & $\begin{array}{l}0,13796 \\
0,0023\end{array}$ & $\begin{array}{l}0,12049 \\
0,0085\end{array}$ & $\begin{array}{l}-0,12907 \\
0,0058\end{array}$ & $\begin{array}{l}0,09582 \\
0,0347\end{array}$ & $\begin{array}{l}0,02091 \\
0,6457\end{array}$ & $\begin{array}{l}0,02250 \\
0,6237\end{array}$ & $\begin{array}{l}0,02650 \\
0,6104\end{array}$ & $\begin{array}{l}0,03319 \\
0,4714\end{array}$ & $\begin{array}{l}0,00291 \\
0,9490\end{array}$ & $\begin{array}{l}0,06852 \\
0,1311 \\
\end{array}$ & $\begin{array}{l}0,11398 \\
0,0131 \\
\end{array}$ & $\begin{array}{l}0,33146 \\
0,0001\end{array}$ & $\begin{array}{l}1,0000 \\
0,0\end{array}$ \\
\hline
\end{tabular}

- Ver no texto o significado das siglas

tervalos de confiança muito amplos, devido à alta concentração de observações na categoria de não risco. Portanto, essas variáveis foram excluídas da análise multivariada.

Quanto à idade da criança, sabemos que as probabilidades de morte e de sobrevivência a cada idade exata (funções qx e px das tábuas de mortalidade) não são lineares na idade ${ }^{27}$.

Foi então necessário buscar uma outra forma funcional que ajustasse melhor a relação entre o "logit" da probalidade de morte, transformação usada em modelos de regressão logísticos, e a idade.

Hosmer e Lemeshow ${ }^{13}$, propõem a inclusão de termos de mais alta ordem da variável idade como uma forma de contornar este problema, sendo a forma quadrática mais apropriada nesse caso.

$\mathrm{Na}$ fase univariada, o modelo quadrático para a idade da criança teve seus coeficientes não significativos, o que pode ser explicado pelo próprio desenho do estudo, onde os controles eram escolhidos de modo pareado aos casos. Entretanto, decidiu-se incluir a idade no modelo multivariado, pois sabe-se que algumas variáveis, tais como desmame, estão associadas à idade da criança, necessitando ser controladas por esta ${ }^{31}$.
Como conclusão da análise univariada, as seguintes variáveis podem ser consideradas, isoladamente, como significativas para explicar a ocorrência da morte por pneumonia: peso ao nascer; idade do desmame; ordem de nascimento da criança; grau de instrução dos pais; número de moradores; renda familiar "per capita"; presença de moradores fumantes; vacinação BCG; história prévia de internacão; história prévia de asma e/ou pneumonia.

A Tabela 5 mostra os resultados obtidos na segunda etapa da análise. Para o Grupo I de variáveis, PESNASC, DESMAME e ORDNASC, mantiveram sua importância na determinação da morte por pneumonia. A não significância da variável "fumo na gestação", nesta fase, deve-se provavelmente à sua associação com as demais do grupo, em particular com o peso ao nascer, indicada pela matriz de correlação (Tabela 3 ).

No grupo dos fatores ligados à condição social da familia (Grupo II), as variáveis que contribuíram para o modelo final foram NMORAD e INSTMAE. O grau de instrução do pai foi excluído desta etapa devido à sua alta correlação com grau de instrução da mãe (Tabela 3) e por ter apresentado grande número de perdas neste quesito.

Ainda neste grupo, vale comentar o coefi- 
Tabela 4. Valores estimados para os coeficientes da regressão logística univariada, risco relativo e intervalos de confiança

\begin{tabular}{|c|c|c|c|c|c|c|c|c|}
\hline \multicolumn{3}{|c|}{ Fatores de risco } & \multirow{2}{*}{$\begin{array}{c}\text { Coefic }^{* *}(1) \\
0,0200\end{array}$} & \multirow{2}{*}{$\frac{D P^{* * *}(2)}{0,1135}$} & \multirow{2}{*}{$\frac{P^{* n+* *}(3)}{0,8604}$} & \multirow{2}{*}{$\frac{R R^{* \cdots+* k}(4)}{1,020}$} & \multicolumn{2}{|c|}{$1 C^{*+\ldots+*+*}(5)$} \\
\hline \multicolumn{2}{|c|}{ Grupo 1} & IDADEC & & & & & 0,817 & 1,274 \\
\hline & & IDADEC2 & $-0,0031$ & 0,0092 & 0,7341 & 0,997 & 0,979 & 1,015 \\
\hline & & PESNASC & $-1,0317$ & 0,1541 & 0,0000 & 0,356 & 0,264 & 0,482 \\
\hline & & -DESMAME & 1,6270 & 0,1685 & 0,0000 & 5,089 & 3,392 & 7,634 \\
\hline & & ORDNASC & 0,8293 & 0,2085 & 0,0001 & 2,292 & 1,523 & 3,448 \\
\hline & & FUMGEST & 0,5460 & 0,1857 & 0,0033 & 1,726 & 1,200 & 2,484 \\
\hline \multicolumn{9}{|c|}{ Grupo II } \\
\hline & & NMORAD & 0,9804 & 0,1917 & 0,0000 & 2,665 & 1,831 & 3,881 \\
\hline & & INSTMAE & 0,6429 & 0,1891 & 0,0007 & 1,902 & 1,313 & 2,755 \\
\hline & & INSTPAI & 0,5808 & 0,2165 & 0,0073 & 1,787 & 1,169 & 2,732 \\
\hline & & PMORFUM & 0,5294 & 0,2140 & 0,0134 & 1,698 & 1,116 & 2,583 \\
\hline & & RENDFPC & $\cdot 0,3633$ & 0,1846 & 0,0491 & 1,438 & 1,001 & 2,065 \\
\hline \multicolumn{9}{|c|}{ Grupo III } \\
\hline & & $\mathrm{BCG}$ & 1,0112 & 0,2061 & 0,0000 & 2,749 & 1,836 & 4,117 \\
\hline & & DOENÇA & 1,4779 & 0,3153 & 0,0000 & 4,383 & 2.362 & 8,132 \\
\hline & & NINTERN & 1,4941 & 0,3370 & 0,0000 & 4,455 & 2,302 & 8,625 \\
\hline • & \multicolumn{8}{|c|}{ Ver no texto o significado das siglas } \\
\hline$*$ & \multicolumn{8}{|c|}{ Coeficiente de regressão } \\
\hline \#** & \multicolumn{8}{|c|}{ Desvio-padrão } \\
\hline 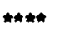 & \multicolumn{8}{|c|}{ Nivel de significância para o coeficiente } \\
\hline 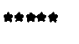 & \multicolumn{8}{|c|}{ Risco relativo aproximado - "Odds ratio" } \\
\hline ... & \multicolumn{8}{|c|}{ Intervalo de confiança para um nivel de significância de $95 \%$. } \\
\hline
\end{tabular}

ciente da variável RENDFPC ( -0.0035 ; N.S.), mostrando com isso que ela perde seu poder de explicação quando controlada pelas demais do grupo; o mesmo acontecendo, de forma menos acentuada, para a variável PMORFUM.

Foi estimado o risco relativo independente de morrer por pneumonia associado com cada variável selecionada na etapa multivariada, e assim estabeleceu-se o modelo final. Somente quatro variáveis são estatisticamente associados com a mortalidade, após o ajuste pelo modelo de regressão logística múltipla: DESMAME, PESNASC, NMORAD e BCG.

Observou-se que todas as variáveis do modelo final têm um risco associado maior do que um, exceto o PESNASC; contudo, para INSTMAE e ORDNASC o limite inferior de seus intervalos inclui o valor um, mostrando com isso que essas variáveis não são relevantes na determinação da morte por pneumonia quando controladas para as outras do modelo final.

A variável idade (sob forma linear e quadrática) foi mantida para controle, apesar de não significante.

A variável "história prévia de doença", do
Grupo III, se incluída no modelo final, teria um coeficiente de 1.7475 ( $p<0,05$ ), com um risco relativo estimado de 5.740 ; todavia, o limite superior do intervalo e o desvio-padrão são muito elevados, o que levou a abandonar esta variável no modelo definitivo.

Conclui-se, então, que os fatores de risco significantes são: idade do desmame, número de moradores e ausência de vacinação com BCG, e o peso ao nascer constituindo-se em fator de proteção.

\section{Discussão}

O primeiro fato que chama a atenção é a importância da variável PESNASC nas 3 etapas da análise. $\mathrm{Na}$ fase multivariada, o PESNASC aparece como a covariável mais significativamente associada ao risco de morrer por pneumonia, enquanto fator de proteção, com um coeficiente de $-1,0460$ no ajuste final. Desde a Investigação Interamericana sobre Mortalidade na Infância ${ }^{29}$, identificou-se o peso ao nascer como o mais importante fator associado à mortalidade e morbidade dos lactentes. Considera-se atualmente não se tratar 
Tabela 5. Valores estimados para os coeficientes da regressão logística multivariada, risco relativo e intervalos de confiança

\begin{tabular}{|c|c|c|c|c|c|c|c|}
\hline \multicolumn{2}{|c|}{ Fatores de risco } & Coefic" (1) & $D^{*+x+2}(2)$ & $P^{*+1+k}(3)$ & $R R^{* t+2+*}(4)$ & & 5) \\
\hline \multicolumn{8}{|c|}{ Grupo 1} \\
\hline & IDADEC & $-0,2815$ & 0,1586 & 0,0776 & 0,755 & 0,553 & 1,030 \\
\hline & IDADEC2 & 0,0213 & 0,0126 & 0,0916 & 1,022 & 0,997 & 1,047 \\
\hline & PESNASC & $-1,0896$ & 0,1708 & 0,0000 & 0,336 & 0,241 & 0,470 \\
\hline & DESMAME & 1,8045 & 0,2449 & 0,0000 & 6,077 & 3,760 & 9,821 \\
\hline & ORDNASC & 0,9764 & 0,2539 & 0,0001 & 2,655 & 1,614 & 4,367 \\
\hline & FUMGEST & 0,1754 & 0,2242 & 0,4341 & 1,192 & 0,768 & 1,849 \\
\hline \multicolumn{8}{|c|}{ Grupo II } \\
\hline & NMORAD & 0,8687 & 0,2032 & 0,0000 & 2,384 & 1,601 & 3,550 \\
\hline & INSTMAE & 0,5523 & 0,2054 & 0,0072 & 1,737 & 1,162 & 2,598 \\
\hline & PMORFUM & 0,3290 & 0,2292 & 0,1512 & 1,390 & 0,887 & 2,177 \\
\hline & RENDFPC & $-0,0035$ & 0,2053 & 0,9864 & 0,997 & 0,666 & 1,490 \\
\hline \multicolumn{8}{|c|}{ Modelo Final } \\
\hline & IDADEC & $-0,2356$ & 0,1691 & 0,1634 & 0,790 & 0,567 & 1,100 \\
\hline & IDADEC2 & 0,0194 & 0,0132 & 0,1433 & 1,020 & 0,993 & 1,046 \\
\hline & PESNASC & $-1,0460$ & 0,1767 & 0,0000 & 0,351 & 0,248 & 0,497 \\
\hline & DESMAME & 1,8329 & 0,2563 & 0,0000 & 6,252 & 3,783 & 10,333 \\
\hline & ORDNASC & 0,4198 & 0,2903 & 0,1489 & 1,522 & 0,861 & 2,688 \\
\hline & INSTMAE & 0,3798 & 0,2439 & 0,1195 & 1,462 & 0,906 & 2,358 \\
\hline & NMORAD & 0,9551 & 0,2560 & 0,0002 & 2,599 & 1,574 & 4,292 \\
\hline & BCG & 0,6873 & 0,2716 & 0,0114 & 1,988 & 1,168 & 3,386 \\
\hline * & \multicolumn{7}{|c|}{ Ver no texto o significado das siglas } \\
\hline$*$ & \multicolumn{7}{|c|}{ Coeficiente de regressão } \\
\hline *** & \multicolumn{7}{|c|}{ Desvio-padrāo } \\
\hline \#*** & \multicolumn{7}{|c|}{ Nivel de significância para o coeficiente } \\
\hline 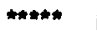 & \multicolumn{7}{|c|}{ Risco relativo aproximado - "Odds ratio" } \\
\hline & \multicolumn{7}{|c|}{ Intervalo de confiança para um nivel de significância de $95 \%$. } \\
\hline
\end{tabular}

apenas de um fator biológico, mas antes, de uma medida-resumo, indicadora, por um lado, dos cuidados pré-natais recebidos e, por outro, das condições de vida da mãe e da família. Victora e col. ${ }^{37}$, com base no estudo longitudinal de Pelotas (RS), demonstrou a estreita relação entre peso ao nascer e veriáveis sociais (renda familiar, escolaridade, hábito de fumar, estado nutricional da mãe, número de consultas pré-natais, entre outros). Os autores evidenciaram um risco relativo de morrer antes de completar um ano por infecções respiratórias, igual a 6,7 para os recém-natos abaixo de $2.500 \mathrm{~g}$, se comparados aos recém-natos acima daquele limiar. A alta correlação entre o baixo peso ao nascer e precárias condições de vida pode ajudar a entender porque as variáveis do Grupo II, relativas ao nível socioeconômico da familia, apesar de serem significativas na fase univariada, diminuem sua significância na segunda fase. Isso levou a um modelo final onde, destas, apenas "número de moradores" permanece estatisticamente signi- ficante. Assim, as variáveis do Grupo II poderiam ser encaradas como variáveis antecedentes e o "peso ao nascer" como variável interveniente, que condensaria aquelas enquanto medida resumo, fazendo com que seu poder de explicação desaparecesse ${ }^{33}$. Deste grupo, permaneceu apenas NMORAD, o que poderia ser explicado pelo fato desta variável indicar maior oportunidade de contatos, facilitando a transmissão das IRAs, que ao se complicarem evoluem para pneumonias. Como já visto, diversos pesquisadores evidenciaram associação entre número de moradores e maior incidência ou letalidade de IRAs ${ }^{1,39}$, o que foi atribuído a uma maior intensidade de exposição, decorrente de maior carga viral. A maior freqüência de IRAs em crianças que permanecem em creches também seria fruto de mecanismos análogos, como já foi observado diversas ve$z^{2}{ }^{2,10}$. Assim, apesar da variável "número de moradores por domicilio" ter sido classificada no presente trabalho como pertencente ao Grupo II, dos fatores socioeconômicos, sua 
permanência no modelo final, após a exclusão de outras variáveis aparentemente mais relevantes, como renda familiar "per capita", por exemplo, seria devida à especificidade biológica do tipo de transmissão nas IRAs, de pessoa - a - pessoa, através dos aerosóis em suspensão no ar, transmissão esta favorecida por uma maior aglomeração domiciliar.

Fica mais fácil, então, entender porque variáveis classicamente consideradas como determinantes da mortalidade infantil, tais como: idade e instrução da mãe, ou renda familiar "per capita", não tenham sido identificadas enquanto fatores de risco no ajuste final.

Por outro lado, algumas particularidades do desenho do estudo podem ajudar a interpretar esses resultados. De fato, o grupo de comparação, os controles sadios, foi pareado socialmente com o grupo de casos, na medida em que foram escolhidos enquanto vizinhos daqueles. Isso levou a uma grande homogeneidade social entre casos e controles, problema já identificado por Post $^{28}$, num estudo anterior com base na mesma investigação.

Apesar disso, foi possível identificar duas outras variáveis como importantes para a mortalidade por pneumonia. Conclui-se ser a "idade do desmame" fator significativo, mesmo se controlada pelo peso ao nascer. $O$ efeito protetor do leite materno na redução da morbimortalidade infantil, em particular por IRAs, tem sido apontado por alguns autores ${ }^{4,11,36,38}$. Entretanto, outros pesquisadores divergem sobre a contribuição da amamentação, que favoreceria apenas a incidência de formas de IRAs mais leves ${ }^{22}$. Barros e col. ${ }^{4}$, com base nos dados levantados pelo estudo prospectivo de Pelotas (RS), discutem a possível existência de um efeito de confusão entre o peso ao nascer e a amamentação, uma vez que os recém-natos de baixo peso tendem a ser desmamados mais precocemente do que os de peso normal. No presente estudo, a variável "idade do desmame" foi categorizada em adequada (se o desmame foi após os 6 meses, ou se a criança mamou até a internaçāo) e não adequada (desmame antes dos 6 meses, ou antes da internação), de modo a contornar os problemas gerados pelo desmame causado pelos óbitos ou internações ocorridos antes dos 6 meses de idade. Assim, esta variável aparece como tendo o maior coeficiente e o maior risco relativo aproximado, no modelo final, mesmo se incluída a variável peso ao nascer, com $\mathrm{p}<$ 0,00001 .

A variável BCG foi a única daquelas per- tencentes ao Grupo III (relativo à utilização dos serviços de saúde) a permanecer no modelo final. Pode-se considerá-la como uma boa "proxy" dos cuidados ministrados à criança. Uma vez que a amostra estudada era composta apenas por óbitos pós-neonatais, e sabendo que o BCG é a primeira vacina obrigatória a ser administrada, em dose única, logo após o nascimento, teoricamente todas as crianças do estudo já deveriam ter sido vacinadas. A "odds ratio" associada ao BCG foi igual a 1,988 e 0 coeficiente foi igual a 0,6873 , com $p<0,0114$.

Embora na fase univariada a "história prévia da doença" e o "número de internações" tenham sido significativos, com $p<0,0001$, sua influência desapareceu após ajuste pelas demais variáveis dos Grupos I e II. Poder-se-ia supor que a vacinação ou não com BCG estaria servindo de condição marcadora do tipo de atenção recebida pela criança. Aquelas que não receberam sequer o BCG muito provavelmente não receberam também as demais vacinas, em especial a antipólio e a tríplice (DPT), que exigem 3 doses, e, portanto, 3 idas ao posto de saúde.

Pode-se supor que, ao adoecer, essas crianças tenham recebido menos cuidados $e$ tenham comparecido ao serviço de saúde mais tardiamente, o que poderia ser comprovado na segunda parte desta investigação, em andamento.

Merece comentátios a exclusão da vatiável relativa à presença de fumantes no domicílio, habitualmente associada a um maior risco de adoecimento por IRAs. Em primeiro lugar, deve ser notada a elevada prevalência do hábito de fumar, nos domicílios visitados. Apenas $19,07 \%$ dos casos e $28,57 \%$ dos controles foram classificados na categoria de nãorisco (nenhum fumante). Por outro lado, os dados disponíveis não permitiam avaliar o grau de exposição (número de cigarros, por exemplo), mas apenas a existência de fumantes, o que pode ter contribuído para o enfraquecimento de uma eventual associação. Além disso, essa variável está associada de forma segnificativa com as demais do grupo.

Os presentes achados são condizentes com os resultados de outro estudo tipo caso-controle desenvolvido no Rio Grande do $\mathrm{Sul}^{38}$, o qual identificou, enquanto fatores de risco para mortalidade por IRAs, o baixo nivel socioeconômico, (que envolve o grau de instrução dos pais), a ausência de amamentação, a falta de suplementação alimentar, a aglomeração domiciliar, o 
número de menores de 5 anos na familia e o baixo peso ao nascer, entre outros. No citado estudo, também não foi constatada uma associação significativa com o fumo dos pais.

Com base nos presentes dados, conclui-se que a mortalidade por pneumonias em menores de um ano está fortemente associada às condições sociais da família, em particular da mãe, sendo esta relação expressa através do baixo peso ao nascer. $O$ desmame precoce também contribui para aumentar o risco de morrer, mesmo levando-se em conta o peso ao nascer. As crianças que vivem em condições de maior aglomeração, refletida no número de moradores por domicílio, estão sujeitas a uma maior exposição aos agentes patogênicos causadores de IRAs, sofrendo maior chance de morrer. As crianças que não recebem sequer os cuidados mínimos, o que é indicado pela não vacinação com $B C G$, apresentam maior risco de vir a falecer por pneumonia.

Em última análise, todos estes fatores parecem estar imbricados, refletindo as condições de vida das famílias, fruto da estrutura social vigente no país.

\section{Agradecimentos}

Aos Professores Kaizô Iwakami Beltrão e Maria Lúcia Fernandes Penna pelas valiosas sugestões e à toda a equipe de coleta, codificação e digitação dos dados.

NIOBEY, F.M.L. et al. [Risk factors for death by pneumonia among children under one year of age in a metropolitan area of Southeastern Brazil. A case-control study]. Rev. Saúde públ., S. Paulo, 26: $229-38,1992$. In a case-control study, a sample of post-neonatal deaths from pneumonia occurring in the Metropolitan Area of Rio de Janeiro, Brazil (1986-1987) were compared with healthy controls who lived in the same neighborhood. Risk factors investigated were variables related to the mother's pregnancy history and the child's birth, to the family's social condition and to the use of health services. Using the univariate logistic regression model, the coefficients of each independent variable, the relative risk and its confidence limits were first estimated. Birth weight and age of weaning were strongly associated with the dependent variable. After adjustment by means of the multiple logistic regression model, only 4 variables remained statistically associated with mortality: age of weaning, birth weight, over crowding, and BCG vaccination. Based on the available data, it was concluded that mortality from pneumonia in children under 1 year of age is significantly related to the social condition of the family, particularly to that of the mother.
Keywords: Pneumonia, mortality. Infant mortality. Risk factors.

\section{Referência Bibliográficas}

1. AABY, P.; BUKH, J.; LISSE I.M.; SMITS, A. Overcrowding and intensive exposure as determinants of meales mortality. Amer.J.Epidem., 120:49-63, 1984.

2. AGRE, F. The relationship of mode of infant feeding and location of care to frequency of infection. Amer.J.Dis. Child, 139: 809-11, 1985.

3. ALLEYNE, G.A.O.; LUELMO, F.; PLAUT, R.; SCHMUNIS, G. Acute respiratory infections in children. Washington, DC, Pan American Health Organization, 1983. (PAHO-Scientific Publication, 493).

4. BARROS, F.C.; VICTORA, C.G.; VAUGHAN, J.P.; SMITH, P.G. Birth-weigth and duration of breast-feeding: are the beneficial effects of human milk being overestimated? Pediatrics, 78: 656-61, 1986.

5. BULLA, A. \& HITZE, K.L Acute respiratory infections: a review. Bull. Whd Hlth Org., 56: 481-98, 1978.

6. CARVALHO, M.L. de; NIOBEY, F.M.L.; VALENTE, J.G. Comparação entre óbitos hospitalares e extra-hospitalares em uma amostra de óbitos infantis pós-neonatais na região metropolitana do Rio de Janeiro - 1986-1987. [Apresentado sob forma de poster ao I Congresso Brasileiro de Epidemiologia, Campinas, SP, 1990].

7. CARVALHO, M.L. de; NIOBEY, F.M.L.; MIRANDA, N.N. de: SABROZA, P.C. Concordância na determinação da causa básica de óbito em menores de um ano na região metropolitana do Rio de Janeiro. Rev.Saúde públ., S.Paulo, 24: 20-7, 1990.

8. CONNOCHIE, K.M. \& ROGHMANN, K.J. Parental smoking, presence of older siblings, and family history of asthma increase risk of bronchiolitis. Amer. J.Dis. Child., 140: 806-12, 1986.

9. DUCHIADE, M.P.; CARVALHO, M.L de; LEAL, M. do C. As mortes "em domicilio" de menores de um ano na região metropolitana do Rio de Janeiro em 1986: um "evento-sentinela" na avaliação dos serviços de saúde. Cadsaúde públ., Rio de Janeiro, 5: 251-63, 1989.

10. FLEMING, D.W.; COCHI, S.L.; HIGHTOWER, A.W.; BROOME, C.U. Childhood upper respiratory tract infections: to what degree is incidence affected by day-care attendance? Pediatrics, 79: 55-60, 1987.

11. FORMAN, M.R; GRAUBARD, B.I.; HOFFMAN, H.J.; BEREN, R; HARLEY, E.E.; BENNETT, P. The PIMA infant feeding study: breast-feeding and respiratory infections during the first year of life. Int.J.Epidem., 13: 447-53, 1984.

12. GARDNER, G.; FRANK, A.L.; TABER, H. Effects of social and family factors on viral respiratory infection and illness in the first year of life. J.Epidem. Communtiy Hlth, 38: 42-8,1984.

13. HOSMER, D.W. \& LEMESHOW, S. Applied logistic regression. Chichester, Willey Interscience, 1989.

14. JAMES, W.J. Longitudinal study of mortidity of dianthoeal and respiratory infections in malnowished children. Amer. J.clin.Nutr., 25: 690-4, 1972.

15. LEOWSKI, J. Mortality from acute respiratory infections in children under 5 years of age: global estimates. Whd Hlth Statist. Quart., 39: 138-44, 1986. 
16. McAULIFFE, J.F.; SOUZA, M.A.; NATIONS, M.K.; SHIELDS, D.S. TAVARES, I.L; LESLIE, J.; ARAUJO, J.G.; GUERRANI, RL. Estudos prospectivos de los efectos de las enfermedades en una comunidad rural del nondeste del Brasil. Bol. Ofic. sanit. panamer, 99: 286-96, 1985.

17. MINISTÉRIO DA SAÚDE. Assisténcia e controle das infecçôes respiratórias agudas (IRA). Brasilia, 1984.

18. MINISTÉRIO DA SAÜDE. Estatísticas de mortalidade: Brasil, 1985. Brasilia, 1988.

19. MOLINA, M.C.B.; GROSS, R.; SCHELL, B.; LEẢO, M.A.C.; STRACK, U.; BRUNKEN, B. Nutritional status of children of low-income communities, Brazil (1986). Rev.Saúde públ., S. Paulo, 23: 89-97, 1989.

20. MONTEIRO, C.A. \& BENICIO, M.H.D · A. Estudo das condições de saúde das crianças do Município de Sảo Paulo, SP (Brasil), 1984/1985. VI - Doença respiratória. Rev. Saúde públ, S.Paulo, 21:380-6, 1987.

21. MOSLEY, W.H. Determinantes biologicos y socioeconomicos de la sobrevivencia en la infancia. Salud publ. Mexico, 30: 312-28, 1988.

22. MYERS, M.G.; FOMON, S.J.; KOONITZ, F.P.; GUINNESS, G.A.; LACHENBRUCH, P.A.; HOLLINGSHEAD, R Respiratory and gastrointestinal illnesses in breast-and formula-fed infants. Amer.J.Dis. Child, 139: 629-32, 1984.

23. NIOBEY, F.M.L.; CASCÃO, A.M.; DUCHIADE, M.P.; SABROZA, P.C. Qualidade do preenchimento de atestados de óbitos de menores de um ano na regiāo metropolitana do Rio de Janeiro. Rev. Saúde públ., S.Paulo, 24: 311-8, 1990.

24. ORGANIZAÇÃO MUNDIAL DA SAÚDE. Manual da classificação estatistica internac:-ial de doenças, lesōes e causas de óbito: revisão 1975. São Paulo, Centro da OMS para Classificação de Doenças em Português, 1980.

25. ORGANIZACION PANAMERICANA DE LA SALUD. Infecciones respiratorias agudas en las Américas. Bol.Epidem. OPS, 1(5): 1-4, 1980.

26. PIO, A.; LEOWSKI, J.; TENDAM, H.G. The magnitude of the problem of acute respiratory infections. In: Douglas, R.M. \& Kerby-Eaton, E., eds. Acute respiratory infections in childhood; Proceedings of an Intemational Workshop, Sydney, 1984. Adelaide, 1985. p. 3-16.

27. POLLARD, A.H.; YUSUF, F.; POLLARD, G.N. Demographic techniques. 2nd ed. Sidney, Pergamon Press, 1974.
28. POST, C. Fatores prognósticos de letalidade hospitalar por diarréia ou pneumonia: um estudo de casos e controles. Rio de Janeiro, 1990. [Dissertaçảo de Mestrado - Escola Nacional de Saúde Pública da FIOCRUZ].

29. PUFFER, R.R. \& SERRANO, C.V. Características de la mortalidad en la nifiez. Washington, Organización Panamericana de la Salud, 1973. (OPAS - Publicación Cientifica, 262).

30. SCHENKER, M.B.; SAMET, J.M.; SPEIZER, F.E. Risk factors for childhood respiratory diseases: the effect of host factors and home environmental exposures. Amer. Rev. resp. Dis., 128: 1038-43, 1983.

31. SCHLESSELMAN, JJ. Case-control studies: desigm, conduct, analysis. Oxford, Oxford University Press, 1982.

32. SERFLING, R.E. Methods for current statistical analysis of excess pneumonia: influenza deaths. Pub. Hlth Rep., 78: 434-506, 1963.

33. SUSSER, M. Causal thinking in the health sciences: concepts and strategies of epidemiology. New York, Oxford University Press, 1973.

34. SZWARCWALD, C.L.; CASTLLHO, E.A.; PINTO, C.B. Características de mortalidade no Estado do Rio de Janeiro, 1977-86. In: Fundação IBGE. Perfil estatístico de crianças e mäes no Brasil: mortalidade infantil e condiçôes de saúde na década de 80. Rio de Janeiro, 1989. p. 91-102.

35. UNICEF. Situação mundial da infância; 1989. Brasília, DF, 1989.

36. VICTORA, C.G.; VAUGHAN, J.P.; LOMBARDI, C.; SMITH, P.G. Evidence for protection by breast-feeding against infant deaths from infections diseases in Brasil. Lancet, 2: 319-21, 1987.

37. VICTORA, C.G.; BARROS, F.C.; VAUGHAN, J.P. Epidemiologia da desigualdade: um estudo longitudinal de 6.000 crianças brasileiras. São Paulo, HUCITEC, 1988.

38. VICTORA, J.P. \& FUCHS, S.C. Risk factors for deaths due to respiratory infections among Brazilian infants. Int.J. Epidem., 18: 918-25, 1989.

39. WRIGTH, G.P. \& WRIGTH, H.L.P. Etiological factors in broncho-pneumonia amongst infants in London. J.Hyg., 44: 15-30, 1945

Recebido para publicaçāo em 24/10/1991 Reapresentado em 11/3/1992 Aprovado para publicação em 18/3/1992 